\title{
Challenges and Lessons Learned from the Department of Reproductive Medicine at Hamad Medical Corporation in Qatar: An Overview of 28 Years of IVF and Future Recommendations
}

\author{
Burjaq $\mathbf{H}^{1 *}$, Khogali FW ${ }^{2}$, Burjaq $\mathbf{R}^{3}$, Al-Bader $\mathbf{M}^{1}$, \\ Abd El Hadi A', Hammoudeh $S^{2}$ \\ ${ }^{1}$ Department of Reproductive Medicine, Women's \\ Hospital, Hamad Medical Corporation, Doha, Qatar \\ ${ }^{2}$ Medical Research Center, Hamad Medical Corporation, \\ Doha, Qatar \\ 3968 ${ }^{\text {th }}$ Street, Doha, Qatar \\ *Corresponding author: Hasan Burjaq, Department \\ of Reproductive Medicine, Women's Hospital, Hamad \\ Medical Corporation, Doha, Qatar
}

Received: August 26, 2021; Accepted: September 30, 2021; Published: October 07, 2021

\begin{abstract}
With Qatar being a pronatalist society, it has been essential to determine the cause of infertility in the country. While a study by Zauner and Girardi has identified several factors that include metabolic diseases, consanguinity, lifestyle, cultural, religious and environmental factors, the doctors and embryologists, based on experience and expertise, at the main and leading Reproductive Medicine Department in the country (DoRM) observed several other factors that result in infertility. Those factors include age, obesity, diabetes, vitamin deficiency and consanguinity. Furthermore, educational, and preventative efforts have been recommended to mitigate the effects of these challenges and to meet the country's pronatalist goals.
\end{abstract}

Keywords: Assisted conception; in vitro fertilization, challenges, infertility, Qatar

\section{Abbreviations}

ACU: Assisted Conception Unit; ART: Assisted Reproductive Technology; DoRM: Department of Reproductive Medicine; HMC: Hamad Medical Corporation; ICSI: Intracytoplasmic Sperm Injection; IVF: In Vitro Fertilization; PCOS: Polycystic Ovarian Syndrome; PGT: Pre-Implantation Genetic Testing; T1DM: Type 1 Diabetes Mellitus; T2DM: Type 2 Diabetes Mellitus

\section{Background}

Qatar, a peninsula in the Arabian Gulf, is a nation with a population exceeding 2.6 million according to the Planning and Statistics Authority as of December 2020 [1]. While millions suffer the burden of infertility globally [2,3], the situation in Qatar is no exception. A recent study has shown a steady decline in fertility rates over the past 50 years, as a result, infertility is considered a national priority [4]. With Qatar being a pronatalist society, determining the causes of infertility is key. Some of the causes previously reported include: high incidence of metabolic disease, consanguinity leading to genetic abnormalities, underlying conditions, lifestyle factors, along with related cultural, religious and environmental factors [4]. However, the main and leading Reproductive Medicine Department in Qatar known as the Department of Reproductive Medicine (DoRM) at Hamad Medical Corporation; previously known as the Assisted Conception Unit (ACU), has observed several other leading causes of infertility in Qatar over the years. Based on the experience of this pioneering institution and its lead embryologists the following factors have been observed and will further be examined in this paper and these include, age, obesity, diabetes, vitamin deficiency, and consanguinity.

\section{Main Challenges}

Main challenges can fall under two main categories: those that are patient related such as medical factors or those that are population based, and those that are related to the unit itself. A study investigated the risk factors of infertility among women in Qatar reported the following factors: maternal age of over 35 years, second hand smoking and weight gain [5]. High rates of infertility, stigmatization, lack of medical staff, absence of fertility centers, and the high cost of health care were among the challenges reported elsewhere [6].

\section{Age and infertility factors}

A recent study published in the committee of the American Society of Reproductive Medicine states that there is a decline in fertility associated with age in both females and males, but predominantly in females [7]. A global burden of diseases study on infertility, which covered 195 countries, reported an increasing trend of infertility since 1990. The standardized female infertility age prevalence rate increased by $15 \%$, while for males the increase was at $8 \%$ for the same period of time [8].

This decline in fertility is seen in women over the age of 35 years due to a decrease in ovarian reserve quantity and oocyte quality $[9,10]$. This is attributed to mitochondrial dysfunction, defects in metabolism, defects in meiosis such as shortening of telomeres leading to chromosomal mis-segregation and hormonal alterations [9]. A retrospective review of 381 cases indicated that ovarian response is a major determinant of success among women ages 40-41 years [11]. In women of childbearing years, who are not using contraception, there has been a steady increase in infertility rates in relation to their age, with a rate of $15 \%$ in women aged $30-34$ and $30 \%$ in women between 
the ages of 35 to 39 . The percentage of women having pregnancies that result in the birth of a child also decreased according to age with the rates being $14-19 \%$ in women aged 35-37 [7]. In fact, an analysis of data taken from 1347 IVF cycles at the DoRM has shown that with a maternal age of 40 and above both the clinical pregnancy rates and the take home baby rates decrease with increasing maternal age, with the clinical pregnancy rate reaching $6.6 \%$ and the take home baby rate reaching zero by the age 49 [12]. With Qatar being a pronatalist society, families are encouraged to have more children, and this often leads women to bear children well past the age of 35. As such, advanced maternal age has been one of the main infertility challenges observed by DoRM doctors and embryologists. It should be noted, however, that research in this area as it relates to Qatar is lacking and future insight is encouraged.

\section{Obesity}

The relationship between obesity and reproductive health continues to be investigated. Dag and Dilbaz have reported that overweight women have a higher incidence of anovulation and menstrual dysfunction, while being an overweight and obese woman has been linked to a higher risk of infertility and subfecundity, miscarriages, and pregnancy complications [13]. Obesity in the Gulf region has been a major and ongoing issue. In fact, one study done in Gulf countries reported an increase in obesity rates due to increased consumption of fast food. Among adults, obesity rates ranged from $2-55 \%$ and $1-30 \%$ among females and males, respectively [14].

Another study observed that women who were obese, scoring higher on the BMI scale, had poor oocyte quality and less uterine receptivity. This was attributed to an increase in inflammatory markers causing directly harmful effects to the oocyte. The mechanism by which this happens however has not been clearly illustrated. In addition to this, side effects from increased adipose tissue results in an increase in androgen release and a decrease estradiol. This androgen/ estradiol ratio mismatch disrupts the process of folliculogenesis resulting in ovarian follicular apoptosis $[15,16]$.

Furthermore, in a systematic review set to identify the effect of obesity on male infertility and how it affects the success rate of assisted reproductive technology, it was found that male obesity contributed to infertility rates. Obese male partners had higher rates of reduced fertility after unprotected sex for 2 years as compared to ideal weight partners. The risk of a non-successful clinical pregnancy from ART was higher in the obese males than in the ideal weight males with a difference of $10 \%$ and an overall decrease in successful live births from ART in obese males as compared to their ideal counterparts. The effects of obesity on sperm function varied but mainly caused a decrease in sperm motility and had a slight effect on sperm concentration, ejaculate volume and morphology of sperm [17].

As obesity is increasing at staggering rates globally, Qatar has also followed in this trend. According to a survey carried out by the Supreme Council of Health (2013) over 70\% of adults in Qatar are overweight or obese [18]. As clearly stated above, a vast association can be seen between obesity and infertility, as such obesity is one of the main challenges observed by health care providers at the DoRM.

\section{Diabetes}

Diabetes impairing female fertility: Diabetes is a known risk factor in the development of female and male infertility. In females, diabetes leads to menstrual irregularities including delayed onset of menarche, absence of menstruation and delayed ovulatory cycles. Women diagnosed with Type 1 Diabetes Mellitus (T1DM) are twice as likely to develop premature menopause compared to women without diabetes, causing a reduction in the reproductive period by at least 6 years [15]. Moreover, it was found in an epidemiologic study conducted by Kjaer and colleagues that a correlation exists between diabetes onset and delays in the menstrual cycle. It was found that diabetic patients, particularly those diagnosed with early onset T1DM prior to the age of ten caused a delay of one year in the age of first menarche as compared to those without diabetes [19].

In a study conducted by Lin and colleagues, it was observed that insulin injections cause hyperinsulinemia by bypassing the liver, and this results in increased levels of circulating androgens and predisposes individuals to develop Polycystic Ovarian Syndrome (PCOS). It was also observed that hyperglycemia and overall poor glycemic control in pregnant patients predisposed them to gestational complications and a decreased birth rate as compared to those pregnant patients without diabetes [20]. Essentially, the hypoglycemia and insulin deficiency that is the result of T1DM leads to a decrease in fertility that is the result of hypogonadism and hyperandrogenism [20]. For patients with Type 2 Diabetes Mellitus (T2DM), the mechanism of infertility is related to indirect risk factors such as obesity, insulin resistance and hyperinsulinemia. All of which cause increased androgen secretion leading to suppressed ovarian function and disrupted ovulatory cycles. PCOS and obesity are also more prevalent in T2DM patients than T1DM; whose negative synergistic effect on endogenous glucose production leads to menstrual and ovarian irregularities [15].

Diabetes impairing male fertility: It is fully acknowledged that there is link between infertility and both T1DM and T2DM, however the mechanism of how this disease affects fertility is not fully understood. One of the reported complications of diabetes is a disturbance in the male reproductive system [21]. In a study aimed to assess the effects of T1DM and T2DM on sperm function and infertility, it was found that in patients with both type 1 and 2 diabetes experienced detrimental effects on sperm function and concentration. This was also proportionally related to how long the patient had the disease. Some of the effects observed include an increased inflammatory response resulting in oxidative stress which leads to minimal sperm vitality and higher rates of DNA fragmentation, damage to the testes, and altered mitochondrial DNA [22].

Males with T1DM had lower progressive sperm motility and lower seminal fluid volume due to delayed epidydimal contraction as compared to T2DM patients. On the other hand, T2DM patients had more sperm DNA fragmentation and loss of sperm functionality due to increased inflammatory processes such as an increase in seminal fluid white blood cells [22].

Overall, both type 1 and 2 diabetes effects female and male fertility rates as mentioned by the proposed mechanisms above. A significantly high incidence of diabetes has been witnessed in Qatar and this is expected to increase overtime. In fact, according to the WHO report (2016), the prevalence of diabetes in Qatar's adult population is $17 \%$ [23]. Another study by and colleagues (2018) found that Qatar has a higher T1DM incidence rate compared to the rest of the world [24]. 
As such, diabetes is one of the main fertility challenges observed in the DoRM. Furthermore, earlier diagnosis of diabetes, strict glycemic control and prevention of diabetic complications can reduce the rates of infertility observed in these patients [22].

\section{Vitamin deficiency}

It has been demonstrated that vitamin deficiency can affect fertility and potentially influence IVF outcomes in infertile couples. The most commonly studied vitamins affecting fertility in males and females are vitamin D and vitamin B12 deficiencies $[25,26]$.

In males, vitamin B12 had positive effects on sperm function. This was observed in rodent studies where rats deficient in vitamin B12 experienced induced alterations in the seminiferous tubules of the testis impairing the spermatogenesis process. This was further established by positive effects with supplementation of vitamin B12 in in-vitro studies. In these studies, supplementation with vitamin B12 increased sperm motility and sperm count. Furthermore, there was reduced oxidative stress damage to sperm, reduced sperm DNA damage, reduced inflammation rates and decreased homocysteine levels. Homocysteine is a biochemical regulated by vitamin B12 and negatively impacts embryo quality in IVF cycles [26].

For vitamin $\mathrm{D}$ deficiency in males, it is hypothesized that changes in testosterone secretion and production can be influenced by osteocalcin, a hormone regulating calcium metabolism in the Leydig cells which is indirectly regulated by vitamin D [25]. In females, vitamin D deficiency plays a role in fertility and influences the outcome of successful IVF cycles. One study observed a positive correlation between serum vitamin D concentration levels and clinical pregnancy rates. The effect of vitamin D was mainly seen on the uterine receptivity and less on ovarian function and embryo development. In women with deficiencies in vitamin D levels <20ng/ $\mathrm{mL}$ there was a reduced chance of successful IVF births and lower clinical pregnancies as compared to women with no deficiencies in vitamin D [27].

Paffoni and colleagues explored the association of vitamin D levels and successful IVF cycles. In this study, two groups of women were observed: those with normal vitamin $\mathrm{D}$ levels and those who were deficient. Oocyte competence, ovarian responsiveness, number of embryos and development were similar between the groups but those with normal vitamin $\mathrm{D}$ levels had slightly higher quality embryos. Clinical pregnancy rates were $20 \%$ and $31 \%$ for the groups, respectively vitamin D serum level of $<20 \mathrm{ng} / \mathrm{mL}$ and $>20 \mathrm{ng} / \mathrm{mL}$ (with baseline vitamin D serum levels being $20 \mathrm{ng} / \mathrm{mL}$. The success rate of embryo implantation was also higher in the normal vitamin D group compared to the deficient group with the best IVF outcomes observed at vitamin $\mathrm{D}$ levels of at least $30 \mathrm{ng} / \mathrm{mL}$ or more. Uterine receptivity was enhanced by vitamin $\mathrm{D}$ supplementation via the regulation of hormone secretion and the effects of these hormones at the uterine level thus enhancing the environment for embryo implantation [28]. While research on the prevalence of vitamin B12 deficiency is lacking in Qatar, a study by Badawi and colleagues shows that the prevalence of vitamin D deficiency in Qatar is as high as 90\% [29]. As such, vitamin deficiency is one of the main challenges observed at the DoRM.

\section{Consanguinity}

Scientists have associated consanguinity with a higher rate of birth defects as well as genetic disorders [30]. One study assessed the prevalence of consanguinity among Qataris and their knowledge of associated risks. Twenty-two percent of participants indicated a cousin relationship between their parents, while $15 \%$ reported that their parents are from the same tribe. Thirty-five percent reported a consanguineous relationship of their own, and $9 \%$ reported an affinal relationship. The authors recommended implementing health strategies that target educating the public as to the effects of consanguineous marriages on future generations [31]. Another study looked at a sample of 1515 participants and found that a consanguinity rate of $54 \%$ was reported. The most common type was the first cousins, at a rate of $35 \%$ [32]. More recently a study investigating the relationship between consanguinity and genetic disorders reported a rate of 20-50\% in the Middle East [30]. A study by Bener and Mohammad investigated the role of consanguinity in a sample of 1228 participants in Qatar. It showed a significantly higher prevalence rate of diabetes mellitus, blood disorders, cancers, mental disorders, asthma, heart disease, gastro-intestinal disorders, hypertension, eye diseases, hearing deficit, and G6PD. Furthermore, the rate of consanguinity among the sample was $43.5 \%$ [33]. In addition, studies have shown that consanguinity affects both male and female infertility via several gene mutations [34,35]. As such this information along with the above studies that clearly indicate that consanguineous marriages are a common and culturally enduring practice, explain why consanguinity is one of the most common main challenges observed at the DoRM.

\section{Recommendations and Future Directions}

Addressing common obstacles or challenges at the population level is one of the recommended approaches, as researchers have highlighted the necessity of challenging the cultural foundation behind such practices as in consanguineous marriages, utilizing targeted programs that address these issues at their social roots [36]. At an international level, a study addressing current issues in assisted reproduction in Europe highlighted that research must be conducted at several levels starting with basic research followed by clinical trials in order to establish new foundations for future procedures [37]. Moreover, several recommendations were outlined in the clinical practice guidelines set by the Reproductive Endocrinology and Infertility Committee, that revolve involve counseling, ovarian reserve testing, oocyte donation, and education [38].

At the local level, the management of risk factors and or chronic diseases through building awareness and early detection may reflect positively on the number and severity of infertility cases [5]. Educational efforts would need to include the younger portion of the population and cover matters pertinent to prevention of disease and promote a healthy lifestyle. While at the family level this would include matters related to fertility and family planning [6]. Knowledge related to age and fertility is another area that can be targeted during educational efforts and campaigns [39]. A Canadian study reported that the time it takes to seek treatment is associated with the degree of fertility awareness, along with the level of education, and ethnicity [40].

Furthermore, a systematic review recommended interventions that target increasing fertility awareness among those with lesser education and among men. The study suggested that these 
interventions be individualized for a stronger effect [41]. The importance of such interventions is illustrated by shaping the decision-making process of involved individuals. Educational efforts based on websites or interactive online tools have been shown to raise awareness [42]. Finally, research into the various aspects related to reproductive medicine and assisted conception is encouraged in order to identify the socio-demographics of the population and aid in building the knowledge necessary to conduct future trials and research activities [43].

\section{Concluding Remarks}

Overall, addressing the obstacles and challenges requires a multi factorial approach taking into consideration the diversity and complexity of these elements. Wide strides towards this have taken place so far at the DoRM at HMC in order to provide IVF services to the Qatari population. The future holds numerous promises with the establishment of the new unit.

\section{References}

1. Planning and Statistics Authority. Monthly Figures on Total Population. 2021

2. Rutstein SO, Shah IH. Infecundity, infertility, and childlessness in developing countries. DHS Comparative Reports No. 9. Calverton, Maryland, USA: ORC Macro. 2004

3. Direkvand-Moghadam A, Sayehmiri K, Delpisheh A, Direkvand-Moghadam A. The global trend of infertility: an original review and meta-analysis. Int J Epidemiol Res. 2014; 1: 35-43.

4. Zauner G, Girardi G. Potential causes of male and female infertility in Qatar. Journal of Reproductive Immunology. Elsevier Ireland Ltd. 2020; 141 103173.

5. Musa S, Osman S. Risk profile of Qatari women treated for infertility in a tertiary hospital: a case-control study. Fertil Res Pract. 2020; 6: 12.

6. Gwet-Bell E, Gwet BB, Akoung N, Fiadjoe MK. The 5 main challenges faced in infertility care in Cameroon. Glob Reprod Heal. 2018; 3.

7. The Practice Committee of the American Society for Reproductive Medicine. Aging and infertility in women. Fertil Steril. 2006; 86: S248-252.

8. Sun $\mathrm{H}$, Gong T-T, Jiang Y-T, Zhang S, Zhao Y-H, Wu Q-J. Global, regional, and national prevalence and disability-adjusted life-years for infertility in 195 countries and territories, 1990-2017: results from a global burden of disease study, 2017. Aging (Albany NY). 2019; 11: 10952-10991.

9. Cimadomo D, Fabozzi G, Vaiarelli A, Ubaldi N, Ubaldi FM, Rienzi L. Impact of Maternal Age on Oocyte and Embryo Competence. Frontiers in Endocrinology. 2018; 9: 327.

10. Ubaldi FM, Cimadomo D, Vaiarelli A, Fabozzi G, Venturella R, Maggiulli $R$, et al. Advanced Maternal Age in IVF: Still a Challenge? The Present and the Future of Its Treatment. Front Endocrinol (Lausanne). 2019; 10: 94.

11. Tsafrir A, Simon A, Revel A, Reubinoff B, Lewin A, Laufer N. Retrospective analysis of 1217 IVF cycles in women aged 40 years and older. Reprod Biomed Online. 2007; 14: 348-355.

12. Alansari L, Khan M, Burjaq H. The Outcome of 1347 IVF Cycles in Women Older Than 40 Year. In RCog Virtual World Congress of the Royal College of Obstetricians \& Gynecologists. 2021.

13. Dağ ZÖ, Dilbaz B. Impact of obesity on infertility in women. J Turkish Ger Gynecol Assoc. 2015; 16: 111-117.

14. ALNohair S. Obesity in gulf countries. Int J Health Sci (Qassim). 2014; 8 : 79-83.

15. Livshits A, Seidman DS. Fertility Issues in Women with Diabetes. Women's Heal. 2009; 5: 701-707.

16. Talmor A, Dunphy B. Female obesity and infertility. Best Pract Res Clin
Obstet Gynaecol. 2015; 29: 498-506.

17. Campbell JM, Lane M, Owens JA, Bakos HW. Paternal obesity negatively affects male fertility and assisted reproduction outcomes: a systematic review and meta-analysis. Reprod Biomed Online. 2015; 31: 593-604.

18. Haj Bakri, A and Al-Thani A. Chronic Disease Risk Factor Surveillance: Qatar STEPS Report 2012. 2013

19. Kjaer K, Hagen C, Sandø SH, Eshøj O. Epidemiology of menarche and menstrual disturbances in an unselected group of women with insulindependent diabetes mellitus compared to controls. J Clin Endocrinol Metab. 1992; 75: 524-529.

20. Lin Y-H, Chen K-J, Peng Y-S, Chen P-C, Yang Y-H. Type 1 diabetes impairs female fertility even before it is diagnosed. Diabetes Res Clin Pract. 2018; 143: 151-158.

21. Ding G-L, Liu Y, Liu M-E, Pan J-X, Guo M-X, Sheng J-Z, et al. The effects of diabetes on male fertility and epigenetic regulation during spermatogenesis. Asian J Androl. 2015; 17: 948-953.

22. Condorelli RA, La Vignera S, Mongioì LM, Alamo A, Calogero AE. Diabetes Mellitus and Infertility: Different Pathophysiological Effects in Type 1 and Type 2 on Sperm Function. Front Endocrinol (Lausanne). 2018; 9: 268.

23. World Health Organization W. Diabetes country profiles-Qatar. 2016.

24. Alyafei F, Soliman A, Alkhalaf F, Sabt A, De Sanctis V, Waseef R, et al. Incidence of type 1 and type 2 diabetes, between 2012-2016, among children and adolescents in Qatar. Acta Biomed Atenei Parm. 2018; 89: 7-10.

25. Cito G, Cocci A, Micelli E, Gabutti A, Russo GI, Coccia ME, et al. Vitamin D and Male Fertility: An Updated Review. World J Mens Health. 2020; 38 : 164-177.

26. Banihani SA. Vitamin B(12) and Semen Quality. Biomolecules. 2017; 7: 42.

27. Pagliardini L, Vigano' P, Molgora M, Persico P, Salonia A, Vailati SH, et al. High Prevalence of Vitamin D Deficiency in Infertile Women Referring for Assisted Reproduction. Nutrients. 2015; 7: 9972-9984.

28. Paffoni A, Ferrari S, Viganò P, Pagliardini L, Papaleo E, Candiani M, et al Vitamin D deficiency and infertility: insights from in vitro fertilization cycles. J Clin Endocrinol Metab. 2014; 99: E2372-2376.

29. Badawi A, Arora P, Sadoun E, Al-Thani A-A, Thani MH Al. Prevalence of vitamin d insufficiency in qatar: a systematic review. J Public health Res. 2012; 1: 229-235.

30. Ben-Omran T, Al Ghanim K, Yavarna T, El Akoum M, Samara M, Chandra $P$, et al. Effects of consanguinity in a cohort of subjects with certain genetic disorders in Qatar. Mol Genet \&amp; genomic Med. 2020; 8: e1051.

31. Sandridge AL, Takeddin J, Al-Kaabi E, Frances Y. Consanguinity in Qatar: knowledge, attitude and practice in a population born between 1946 and 1991. J Biosoc Sci. 2010; 42: 59-82.

32. Bener A, Alali KA. Consanguineous marriage in a newly developed country: the Qatari population. J Biosoc Sci. 2006; 38: 239-246.

33. Bener A, Mohammad RR. Global distribution of consanguinity and their impact on complex diseases: Genetic disorders from an endogamous population. Egypt J Med Hum Genet. 2017; 18: 315-320.

34. Feng R, Sang Q, Kuang Y, Sun X, Yan Z, Zhang S, et al. Mutations in TUBB8 and Human Oocyte Meiotic Arrest. N Engl J Med. 2016; 374: 223-232.

35. Lawrenz B, Coughlan C, Melado L, Fatemi HM. Ethnical and sociocultura differences causing infertility are poorly understood-insights from the Arabian perspective. J Assist Reprod Genet. 2019; 36: 661-665.

36. Kaltham Ali A-G. Consanguineous marriage in Qatar: Marriage selection in a society in transition. 2012; 2012.

37. Harper JC, Geraedts J, Borry P, Cornel MC, Dondorp W, Gianaroli L, et al. Current issues in medically assisted reproduction and genetics in Europe: research, clinical practice, ethics, legal issues and policy. European Society of Human Genetics and European Society of Human Reproduction and Embryology. Eur J Hum Genet. 2013; 21: S1-21. 
38. Liu K, Case A, Cheung AP, Sierra S, AlAsiri S, Carranza-Mamane B, et al. Advanced Reproductive Age and Fertility. J Obstet Gynaecol Canada. 2011; 33: $1165-1175$

39. Deatsman S, Vasilopoulos T, Rhoton-Vlasak A. Age and Fertility: A Study on Patient Awareness. JBRA Assist Reprod. 2016; 20: 99-106.

40. Swift BE, Liu KE. The effect of age, ethnicity, and level of education on fertility awareness and duration of infertility. J Obstet Gynaecol Canada. 2014; 36: 990-996.

41. Pedro J, Brandão T, Schmidt L, Costa ME, Martins MV. What do people know about fertility? A systematic review on fertility awareness and its associated factors. Ups J Med Sci. 2018; 123: 71-81.

42. Delbaere I, Verbiest S, Tydén T. Knowledge about the impact of age on fertility: a brief review. Ups J Med Sci. 2020; 125: 167-174.

43. Burjaq H, Al-Bader M, Shahata M. The Assisted Conception Unit in Hamad Medical Corporation: An Overview, Achievements, and Future Directions. J Pregnancy Newborn care. 2020; 3. 